\title{
Research on user preference acquisition method based on decision matrix
}

\author{
Huiran Zhao ${ }^{1}$, Lei Shi ${ }^{1}$, WeijieYao ${ }^{1}$,YuexingZhong ${ }^{1}$ \\ 1School of Computer Engineering,City Institude,Dalian University of Technology,dalian \\ 116600,China \\ email: zhr2006@dlut.edu.cn
}

key words: User preference;Decision matrix;

\begin{abstract}
The main purpose of this paper is to study the decision matrix of user preference data acquisition theory and method based on, to establish effective user preference acquisition model and the method, to further enrich and improve user preference decision matrix theory and method and for the user decision support system provides the decision data analysis method
\end{abstract}

Decision is to solve a problem or to achieve certain goals is proposed to solve problems and achieve targets for a variety of feasible scheme, with the help of some tools, technology and information, according to some evaluation criteria and standards, in a variety of options, select an optimal or satisfactory case analysis, judgment and put into the implementation of the management process. With the development of the Internet, users get more and more information from the Internet, at the same time, users also publish their own information on the Internet to provide to other users. "New Moore's law" points out that the information on the Internet is increasing at a rate of six months each month. The increase in the amount of information should be given to the Internet users to bring more convenience, But that is not the case, in the face of vast information, It takes a lot of time to find the information you need to take a lot of time to search for the information you need.Users often get lost in the vast amount of information available on the web.At the same time, in accordance with the requirements of a certain part of the user has lost contact with these valuable users, network application providers have gradually come to understand this problem. How to learn the user's interest in the Internet needs and can provide users with accurate information to become the key to attract users and expand the number of users.

Under this kind of background, researchers have carried out a lot of research in two directions of data mining and topic search. From the Amazon, Taobao and other e-commerce provider of user interest mining, the Internet Movie Database and other large public database project on the user's preference to other information service website through all aspects of the user's information mining user preferences and the user provides accurate and personalized service.

Therefore, user preference mining based on decision matrix is very important, and the research of decision matrix is implemented by mathematical model and behavior aggregation model. Among them, the mathematical model is based on economics and operations research, and the individual preference quantity aggregation algorithm is studied by mathematical method. Behavior aggregation model based on social psychology and organizational behavior, The effect of group interaction on preference aggregation was observed and analyzed by experimental methods. Decision making is often based on their own professional knowledge and experience given by the judgment information, these judgments are usually expressed through the decision matrix. Therefore, the decision matrix has become a powerful tool and means to deal with the problem of user preference mining. 


\section{Matters needing attention on Decision matrix acquisition decision information}

For the qualitative evaluation criteria or alternative plan, usually by pairwise comparison technique score, and then construct the pairwise comparison decision matrix and decision matrix for short, finally based on decision matrix to calculate the weight and ranking. Decision matrix is an effective form of user preference information.The comparison technology is also widely used in the real decision problems such as voting system, intelligent system and game project. It plays an irreplaceable role in enriching and developing the modern decision technology.

In order to construct an effective decision matrix, we must do a good job of collecting and making decision information, and should pay attention to the following points:

(1)Rational choice of multiple point decision information source. As far as possible, choose the right decision information sources, and truly play the authenticity of the information source and the key. In the process of statistics, according to different criteria, different information sources are required to complete the judgment matrix, rather than just a source of information to complete all the decision matrix.

(2)Create a good environment for information statistics. On the one hand, to prepare the necessary and reliable data and information for the decision matrix, including decision rules, on the other hand, to create a suitable database design for the decision data.

(3)Master the correct information collection method. Before the information collection to be designed to be clear and easy to fill in the information table. In the process of information collection, the decision matrix is allowed to determine the violation of the transfer and consistency, and the decision matrix is also allowed to judge whether the problem is not familiar or unclear.In addition to the use of a direct hit, in principle, should adhere to the two two paired comparison of the decision-making method, in order to improve the credibility of the decision matrix.

(4)Analyze data and information in time and feedback if necessary.Decision matrix for the collection of information to be properly analyzed, when a variety of reasons for the apparent failure to judge or different decision matrix judgments have a great difference, should be feedback, and then the next analysis. Finally, a reasonable result is obtained.

\section{Method for constructing decision matrix}

In order to obtain reliable individual decision matrix, the first measure opinions and decisions of concentration or dispersion and said the decision preference decision matrix to optimize the processing, from the point of view of mathematical statistics choice commonly used metrics are as follows:

Set $X=\{x 1, x 2, \ldots X r\}$ is the observation sample for $R$ decision information, Vector $(1,2, \ldots R()$ is a vector of the importance of the decision data, which, $=1, \mathrm{i}>0,(\mathrm{i}=1,2), \ldots \mathrm{R})$.

(1)Weighted mean value: $\mathrm{U}(\mathrm{X})=\sum_{i=1}^{r} \lambda_{i} X_{i}$

$\mathrm{U}(\mathrm{X})$ indicates the average level of evaluation value of decision information;

(2)standard deviation: $\sigma(X)=\sqrt{\sum_{i=1}^{r} \lambda_{i}\left(X_{i}-U(X)\right)^{2}}$

$\sigma(X)$ is the volatility of expert evaluation value;

(3) Reliability:

$$
\psi(X)=\min _{i} \int_{-\infty}^{x_{i}} \frac{1}{\sqrt{2 \pi} \sigma} \exp \left[-\frac{(t-a)^{2}}{2 \sigma^{2}}\right] d t
$$

$\Psi(X)$ is the level of credibility of group opinions;

(4) kurtosis: $E_{4}(X)=\sum_{i=1}^{r} \lambda_{i}\left(x_{i}-U(X)\right)^{4} / \sigma(X)^{4}-3$ (1-4)

$E_{4}(X)$ to express the consistency of expert opinion;

(5)skewness: $S_{3}(X)=\sum_{i=1}^{r} \lambda_{i}\left(x_{i}-U(X)\right)^{3} / \sigma(X)^{3}$ (1-5)

$S_{3}(X)$ : representation of the decision expert opinion on the weighted mean value of symmetry;

(6) variationcoefficient: $V(X)=\sigma(X) / U(X)$ 
$V(X)$ indicates the coordination of the value of group evaluation.

In the process of user preference information collection, the individual preference given by the decision matrix must satisfy the given consistency, coordination, reliability and so on. Among them, the kurtosis index can control the consistency of group opinion; coordination requirements variation coefficient is less than or equal to the predetermined coefficient of variation; reliability requirements aggregation result is greater than or equal to the predetermined reliability standard.

\section{The basic method of user preference collection}

In the research of practical decision matrix, the method of user preference acquisition based on decision matrix has been widely used. The weight vector obtained by the user's preference judgment directly determines the order of the decision matrix. At present, there are two kinds of acquisition methods which are widely used in the acquisition of user preference based on decision matrix.

A decision matrix consisting of $r(r \geq 2)$ decision information is assumed. In a single standard, the first pair of alternatives were paired comparison, and then collected individual members' preferences for a single group preferences, and finally according to the group preference for alternatives to sort. Specifically, according to the given criteria, this $\mathrm{n}$ alternative to match the two two comparison, Each individual decision maker is given a judgment matrix:

$A^{(k)}=\left(a_{i j}^{(k)}\right)_{n \times n}(k=1,2, \cdots, r)$

Among them, $a_{i j}^{k}$ represents the element of the decision matrix given by the first $\mathrm{k}$ decision information. Individual weight vectors based on these individual decision matrices are recorded as:

$$
W^{(k)}=\left(w_{1}^{(k)}, w_{2}^{(k)}, \cdots, w_{n}^{(k)}\right)^{T}(k=1,2, \cdots, r)
$$

Group weight vector is obtained by group member preference:

$$
W^{G}=\left(w_{1}^{G}, w_{2}^{G}, \cdots, w_{n}^{G}\right)^{T}
$$

In addition, the assumption that the importance of $\mathrm{R}$ decision makers of the weight vector is ( $\lambda 1$, $\lambda 2, \ldots \lambda \mathrm{r}), \sum_{i=1}^{r} \lambda_{i}=1, \lambda \mathrm{i}>0, \quad(\mathrm{i}=1,2, \ldots, \mathrm{r})$.

Most of the information collection methods do not consider the difference of the credibility of the decision matrix, which is determined by the decision information. That is to say, no consideration is given to the weight of the important degree of the decision information.Weighted Geometric Mean Method (WGMM) is used to obtain the weight of the important degree of the decision information.This method has a better consistency with the weighted geometric mean of the sampling method.

\section{Evaluate the effect of user preference acquisition method}

In order to evaluate the effect of the group preference aggregation method, two compatibility indexes are introduced to measure the compatibility between the individual decision matrix and the group weight vector. Two important compatibility indexes are geometric compatibility index (GCI) and priority deviation quantity index (PVN), which are defined as follows: 


\section{Geometrical compatibility index(GCI)}

Set $\mathrm{WG}=\left(W_{1}^{G}, W_{2}^{G}, \ldots, W_{n}^{G}\right) \mathrm{T}$ is the group weight vector obtained by a kind of aggregation model.The geometric compatibility index $(G C I)$ between the individual decision matrix $A^{(k)}=\left(a_{i j}^{(k)}\right)_{n \times n}(k=1,2, \cdots, r)$ and the group weight vector is $G C I=\sum_{k=1}^{r} \lambda_{k}\left(\frac{2}{(n-2)(n-1)} \sum_{i<j} \ln ^{2}\left(a_{i j}^{(k)} w_{j}^{G} / w_{i}^{G}\right)\right)$. (1-10)

Among them, $\lambda \mathrm{k}$ is the weight of individual decision-making information, meet $\sum_{k=1}^{r} \lambda_{k}-1, \lambda_{k}>0(k-1,2, \cdots, r)$.

\section{Priority departure from quantitative indicators $(P V N)$}

$$
\begin{aligned}
& P V N=\sum_{k=1}^{r} \frac{2}{(n-2)(n-1)} \sum_{i<j} I_{i j}\left(A^{(k)}\right) \\
& I_{i j}\left(A^{(k)}\right)=\left\{\begin{array}{cc}
1 & \omega_{i}^{G}<\omega_{j}^{G}, a_{i j}>1 \\
0.5 & \omega_{i}^{G}=\omega_{j}^{G}, a_{j i} \neq 1 \\
0.5 & \omega_{i}^{G} \neq \omega_{j}^{G}, a_{j i}=1 \\
0 & \text { other }
\end{array}\right.
\end{aligned}
$$

In fact, the geometrical compatibility index $(G C I)$ is the basic compatibility index, and the priority deviation quantity index $(P V N)$ is the ordinal compatibility index.The compatibility between the individual decision matrix and the group weight vector is measured by the compatibility index to evaluate the effect and advantage of the proposed method.

\section{Summary}

The main purpose of this paper is to study the decision matrix of user preference data acquisition theory and method based on, to establish effective user preference acquisition model and the method, to further enrich and improve user preference decision matrix theory and method and for the user decision support system provides the decision data analysis method. The decision rule conflict, decision-making information analysis limitations, the policy-making goal is not to be measured and contradiction and making itself complexity, and many other factors, decision matrix often don't have exactly the same even satisfactory consistency. Decision matrix with satisfactory consistency is the precondition and basic requirement of decision making.Therefore, the first issue of user preference acquisition is how to establish the consistency test and inspection criteria of the decision matrix, and to test the consistency of the decision matrix.First of all, the definition, properties and theorems of the decision matrix and its consistency are briefly summarized.Then, it introduces the definition and expression of the consistency index of decision matrix.Finally, a numerical example is used to illustrate the validity and the validity of the statistical method for the consistency check of the decision matrix.Through the research on the consistency test of the decision matrix, the flexibility and practicality of the consistency checking of the decision matrix are improved. 


\section{Reference}

[1]Ergu D,Kou G,Peng Y,Shi Y. A Simple Method to Improve the Consistency Ratio of the Pair-wise Comparison Matrix in ANP [J]. European Journal of Operational Research. 2011,213(1): $246-259$.

[2]Dong Y C, Zhang G Q, Hong W C, Xu Y F. Consensus models for AHP group decision making under row geometric mean prioritization method [J]. Decision Support Systems, 2010, 49(3): $281-289$.

[3]Srdjevic B,Srdjevic Z. Synthesis of individual best local priority vectors in AHP-group decision making [J]. Applied Soft Computing, 2013, 13(4): 2045 - 2056.

[4]Daniel Kikuti, Fabio Gagliardi Cozman, Ricardo Shirota Filho, SequentialDecision Making with Partially Ordered Preferences, ArtificialIntelligence (2010).

[5]Shui-li Huang, Comparision of Utility-Based Recommendation Methods, PACIS 2008. 